\title{
Electrocardiographic findings and frequency of arrhythmias in Bartter's syndrome
}

\author{
CARINA BLOMSTRÖM-LUNDQVIST, * KENNETH CAIDAHL, $\dagger$ \\ S BERTIL OLSSON, * AVARUDIN
}

From the Medical Department I and II, Division of *Cardiology and $\ddagger$ Endocrinology, and $\dagger$ Department of Clinical Physiology, Sahlgren's Hospital, Gothenburg, Sweden

SUMMARY Twenty four hour electrocardiograms in 20 patients with Bartter's syndrome, a $\stackrel{9}{-}$ disorder associated with chronic potassium deficiency, were analysed for atrial and ventricular $i$ extrasystoles, pauses (RR interval $>2 \mathrm{~s}$ ), and heart rate. The 12 lead resting electrocardiogram was $\not$ also evaluated. There were slight electrocardiographic changes with ST segment depression ( $\geqslant-$ 을 $0.5 \mathrm{~mm})$ in seven patients, flat or low amplitude $T$ waves in seven, and $U$ waves $(\geqslant+1.0 \mathrm{~mm})$ in three patients. The QT interval was prolonged in 18 patients. Nine patients had one or more 3 ventricular extrasystoles in 24 hours. Only two patients had more than 200 ventricular $\frac{\widehat{O}}{\mathrm{~S}}$ extrasystoles in 24 hours. No patient had ventricular tachycardia. A total of nine patients had one or $\vec{\bullet}$ more atrial extrasystoles in 24 hours, but only one patient had more than 200 in 24 hours. One 0 patient had an attack of non-sustained supraventricular tachycardia. No patient had pauses.

Dangerous tachycardia was rare in these patients with chronic potassium deficiency caused by Bartter's syndrome. The general pattern of slight electrocardiographic changes may reflect an adaptation of the myocardium to hypokalaemia. Further studies are, however, needed to determine whether these findings are relevant to long term prognosis.

Potassium is responsible for most electrolyte induced disturbances of cardiac rhythm. Although several studies have shown an increase in potentially dangerous ventricular arrhythmias in patients with hypokalaemia, ${ }^{1-3}$ the subject is still highly controversial. ${ }^{45}$ The cause and effect relation is obscured by inadequate study techniques and by the tendency for arrhythmia associated with the underlying diseases.

Studies with microelectrodes in rabbits showed that alterations in potassium concentrations may cause significant changes in depolarisation, repolarisation, and pacemaker activity in various cardiac cells.$^{67} \mathrm{~A}$ decrease in extracellular potassium concentrations results in a more negative resting membrane potential, ${ }^{8-10}$ a prolonged action potential duration, ${ }^{6811}$ and an increased slope of spontaneous diastolic depolarisation. ${ }^{11}$ Theoretically, these changes could lead to increased automaticity, ${ }^{11}$ slowed conduction, and/or increased dispersion of repolar-

Requests for reprints to Dr Carina Blomström-Lundqvist, Division of Cardiology, Medical Department I, Sahlgren's Hospital, S-413 45 Gothenberg, Sweden.

Accepted for publication 20 September 1988 isation and refractoriness, ${ }^{11}$ which may lead to tachycardia as well as to various types of conduction disturbances. ${ }^{12-14}$ There are not many studies of the electrophysiological effects of magnesium and its interaction with potassium. ${ }^{15}$

Several studies reported a correlation between the surface electrocardiogram and changes in the action potential resulting from alterations in potassium $\delta$ concentrations. ${ }^{69}$ Although the surface electrocar- $₹$ diogram does not give precise information on the ? electrical behaviour of the cell, it represents the sum $D$ of all depolarisation and repolarisation of the myocardial fibres and has therefore been used to assess $N$ disturbances in electrolyte balance.

Bartter's syndrome is characterised by renal $\mathcal{O}$ potassium wasting, hypokalaemic alkalosis, hyper- $N$ reninaemia, and normal blood pressure. ${ }^{16}{ }^{17}$ Hypomagnesaemia is also common. ${ }^{18}$ The primary defect is thought to be a renal tubular dysfunction. Bartter's syndrome is a rare hereditary disorder that $\stackrel{\oplus}{+}$ is often recognised by the incidental finding of 0 chronic hypokalaemia in an otherwise apparently

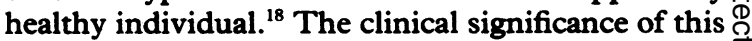
chronic electrolyte disturbance is unknown. We have $\stackrel{\mathbb{Q}}{\mathbb{Q}}$ assessed the prevalence of atrial and ventricular 
arrhythmias and have critically evaluated the electrocardiographic changes in these patients.

\section{Patients and methods}

\section{PATIENTS}

We studied 20 patients (seven men and 13 women) in whom Bartter's syndrome was diagnosed from 1972 to 1988 . The mean age at the time of the study was 44.3 years (range 24 to 69 years). All patients showed renal potassium wasting with hypokalaemia, hyperreninaemia, high or high normal urinary aldosterone excretion, and they were all normotensive. Other causes of potassium wasting - that is, renal tubular acidosis, vomiting, or abuse of diureticshad been excluded. The plasma concentration of bicarbonate was raised or high normal, urinary chloride excretion was normal, and repeated screening for diuretics in urine was negative in all patients. No patient was taking cardioactive drugs. The long term follow up of 19 of these 20 patients has been reported elsewhere. ${ }^{18}$ Eleven patients have also been examined by bicycle ergometer stress testing and cross sectional echocardiography. Of the eleven patients who underwent an exercise test, all except one reached the predicted maximal workload and pulse rate (unpublished observations). Table 1 summarises the clinical details.

\section{METHODS}

All patients had a physical examination, a 12 lead resting electrocardiogram, and 24 hour ambulatory
Holter monitoring. Blood samples for the analysis of serum potassium, magnesium, and calcium were obtained at the time of the studies.

\section{Electrocardiogram}

The electrocardiograms were recorded on paper at a paper speed of $50 \mathrm{~mm} / \mathrm{s}$ on a six channel Mingograph (Siemens Elema 82) for the analysis of $P Q$ intervals, QRST intervals, deviations in ST segments, and amplitude of $Q R S$ complexes, $T$ waves, and $U$ waves. One $\mathrm{mV}$ corresponded to $10 \mathrm{~mm}$. All measurements were made in leads II and V4. Each electrocardiogram was also interpreted according to the Minnesota code. ${ }^{19}$ The QT interval was defined as the time from QRS onset to $(a)$ the end of the $T$ wave (QTE) and (b) the point at which the tangent of the T wave descent crossed the baseline (QT tan) ${ }^{20}$ When the end of the $T$ wave was obscured by an $U$ wave, the $T$ wave offset was taken as the time of intersection of the $\mathrm{T}$ and $\mathrm{U}$ wave. ${ }^{21} \mathrm{QT}$ measurements were corrected for heart rate (QTc) by Bazett's formula. ${ }^{22}$ The upper limit of normal of QTc was defined as $0.425 \mathrm{~s}$ for men and $0.44 \mathrm{~s}$ for women. ${ }^{22}$ The mean value of three consecutive measurements was used in the analysis.

The electrocardiographic patterns were classified as showing changes that were "typical" of or "compatible" with hypokalaemia or "no significant changes", as described by Surawicz et al..$^{23}$ Classification was based on: (a) ST segment depressions $\geq$ $0.5 \mathrm{~mm},(b) U$ wave amplitude $>+1 \mathrm{~mm}$, and $(c) U$ wave amplitude larger than $T$ wave amplitude in the

Table 1 Clinical and laboratory data and electrocardiographic measurements in 20 patients with Bartter's syndrome

\begin{tabular}{|c|c|c|c|c|c|c|c|c|c|c|c|c|c|c|c|}
\hline \multirow[b]{2}{*}{$\begin{array}{l}\text { Case } \\
\text { No }\end{array}$} & \multirow[b]{2}{*}{$\begin{array}{l}\text { Age (yr) } \\
\text { and sex }\end{array}$} & \multicolumn{2}{|l|}{ Serum } & \multirow[b]{2}{*}{$P Q$} & \multirow[b]{2}{*}{$Q R S$} & \multicolumn{2}{|c|}{$Q T E^{\star}$} & \multicolumn{2}{|c|}{$Q T$ tan $\star$} & \multicolumn{2}{|l|}{$S T$} & \multicolumn{2}{|c|}{$T$ wave } & \multicolumn{2}{|c|}{ U wave } \\
\hline & & $\begin{array}{l}K \\
(\mathrm{mmol} / l)\end{array}$ & $\begin{array}{l}\mathrm{Mg} \\
(\mathrm{mmol} / \mathrm{l})\end{array}$ & & & $I I$ & $V 4$ & $I I$ & $V 4$ & $I I$ & $V 4$ & $I I$ & $V 4$ & $I I$ & $V 4$ \\
\hline $\begin{array}{r}1 \\
2 \\
3 \\
4 \\
5 \\
6 \\
7 \\
8 \\
9 \\
10 \\
11 \\
12 \\
13 \\
14 \\
15 \\
16 \\
17 \\
18 \\
19 \\
20\end{array}$ & $\begin{array}{l}46 \mathrm{~F} \\
38 \mathrm{~F} \\
59 \mathrm{~F} \\
27 \mathrm{~F} \\
44 \mathrm{~F} \\
38 \mathrm{~F} \\
24 \mathrm{~F} \\
66 \mathrm{~F} \\
43 \mathrm{~F} \\
40 \mathrm{~F} \\
43 \mathrm{~F} \\
34 \mathrm{~F} \\
34 \mathrm{~F} \\
36 \mathrm{M} \\
66 \mathrm{M} \\
45 \mathrm{M} \\
35 \mathrm{M} \\
54 \mathrm{M} \\
28 \mathrm{M} \\
69 \mathrm{M}\end{array}$ & $\begin{array}{l}2 \cdot 8 \\
2 \cdot 3 \\
2 \cdot 4 \\
3 \cdot 0 \\
2 \cdot 4 \\
2 \cdot 9 \\
2 \cdot 9 \\
2 \cdot 8 \\
3 \cdot 1 \\
3 \cdot 3 \\
2 \cdot 3 \\
3 \cdot 0 \\
2 \cdot 9 \\
2 \cdot 8 \\
2 \cdot 7 \\
2 \cdot 4 \\
2 \cdot 7 \\
2 \cdot 8 \\
2 \cdot 4 \\
2 \cdot 5\end{array}$ & $\begin{array}{l}0.5 \\
0.5 \\
0.5 \\
0.8 \\
0.6 \\
0.7 \\
0.5 \\
0.4 \\
0.6 \\
0.8 \\
0.7 \\
0.7 \\
0.5 \\
0.6 \\
0.6 \\
0.6 \\
0.6 \\
0.5 \\
0.5 \\
0.6\end{array}$ & $\begin{array}{l}0.18 \\
0.15 \\
0.18 \\
0.14 \\
0.14 \\
0.14 \\
0.15 \\
0.15 \\
0.11 \\
0.19 \\
0.17 \\
0.15 \\
0.14 \\
0.16 \\
0.18 \\
0.16 \\
0.19 \\
0.19 \\
0.14 \\
0.16\end{array}$ & $\begin{array}{l}0.11 \\
0.09 \\
0.07 \\
0.08 \\
0.10 \\
0.09 \\
0.10 \\
0.08 \\
0.09 \\
0.08 \\
0.12 \\
0.11 \\
0.10 \\
0.11 \\
0.08 \\
0.10 \\
0.09 \\
0.09 \\
0.09 \\
0.10\end{array}$ & $\begin{array}{l}0.46 \\
0.45 \\
0.45 \\
0.40 \\
0.48 \\
0.45 \\
0.42 \\
0.47 \\
0.46 \\
0.49 \\
0.47 \\
0.47 \\
0.48 \\
0.42 \\
0.62 \\
0.45 \\
0.45 \\
0.45\end{array}$ & $\begin{array}{l}0.42 \\
0.45 \\
0.45 \\
0.41 \\
0.47 \\
0.44 \\
0.43 \\
0.49 \\
0.48 \\
0.47 \\
0.48 \\
0.47 \\
0.46 \\
0.48 \\
0.44 \\
0.41 \\
0.62 \\
0.51 \\
0.43 \\
0.46\end{array}$ & $\begin{array}{l}0.44 \\
0.46 \\
0.45 \\
0.39 \\
0.47 \\
0.43 \\
0.40 \\
0.47 \\
0.46 \\
0.46 \\
0.45 \\
0.52 \\
0.45 \\
0.52 \\
0.49 \\
0.48 \\
0.46\end{array}$ & $\begin{array}{l}0.41 \\
0.43 \\
0.48 \\
0.41 \\
0.46 \\
0.43 \\
0.41 \\
0.53 \\
0.47 \\
0.45 \\
0.44 \\
0.44 \\
0.49 \\
0.47 \\
0.43 \\
0.53 \\
0.48 \\
0.42 \\
0.46\end{array}$ & $\begin{array}{c}0 \cdot 5 \\
-0 \cdot 7 \\
0 \\
0 \\
-0 \cdot 3 \\
-0 \cdot 5 \\
0 \\
-1 \cdot 0 \\
-0 \cdot 3 \\
0 \\
0 \\
0 \\
-0 \cdot 3 \\
-0 \cdot 5 \\
0 \\
0 \\
0 \\
0 \\
0 \\
0\end{array}$ & $\begin{array}{c}0 \\
-0.5 \\
0 \\
0.5 \\
-0.4 \\
-0.4 \\
0.5 \\
-0.5 \\
-0.2 \\
0 \\
0.5 \\
0 \\
0 \\
-1.0 \\
-0.5 \\
0.5 \\
0 \\
0 \\
0 \\
-0.6\end{array}$ & $\begin{array}{l}3.0 \\
2.2 \\
1.2 \\
3.7 \\
2.5 \\
3.0 \\
3.5 \\
1.0 \\
1.5 \\
2.0 \\
4.0 \\
0 \\
1.2 \\
1.8 \\
0.5 \\
2.5 \\
4.5 \\
3.0 \\
2.0 \\
2.5\end{array}$ & $\begin{array}{l}2.3 \\
2.7 \\
0.8 \\
3.2 \\
2.8 \\
2.5 \\
4.8 \\
1.3 \\
1.5 \\
2.0 \\
4.2 \\
2.5 \\
1.3 \\
0.5 \\
3.0 \\
8.0 \\
4.8 \\
3.3 \\
2.5\end{array}$ & $\begin{array}{l}0.4 \\
0.7 \\
0.3 \\
0.7 \\
0.6 \\
0.5 \\
0.1 \\
0.4 \\
0.7 \\
0.8 \\
0.3 \\
0.4 \\
0.8 \\
0.5 \\
1.2 \\
1.1 \\
1.0 \\
0.9\end{array}$ & $\begin{array}{l}0.4 \\
0.7 \\
0.3 \\
0.7 \\
0.7 \\
0.4 \\
0.2 \\
- \\
0.3 \\
0.4 \\
0.3 \\
0.5 \\
0.5 \\
0.5 \\
0.5 \\
1.1 \\
1.2 \\
0.8 \\
0.9\end{array}$ \\
\hline
\end{tabular}

$\star$ Corrected for heart rate.

Normal limits of serum concentrations of potassium and magnesium at our laboratory are 3.6-4.5 mmol/1 and $0.7-1 \cdot 2 \mathrm{mmol} / \mathrm{l}$ respectively. Intervals are given in seconds. ST deviations and $T$ and $U$ wave amplitudes are given in millimetres. $K$, potassium; $M g$, magnesium. 
same lead. The electrocardiogram was classified as "typical" if three or more features were present in the two leads and as "compatible" if two features or one of the features related to the $U$ wave were present. ${ }^{8}$

\section{Holter monitoring}

Ambulatory electrocardiographic recordings were obtained on two-channel 24 hour portable tape recorders (Reynolds Medilog) while the patients followed their daily activities. Each patient was asked to keep a diary of all activities during the recording. Tapes were analysed by a semiautomatic arrhythmia analyser (Reynolds Pathfinder) ${ }^{24}$ operated by an experienced technician who was supervised by C B-L.

Venticular extrasystoles were validated by the operator and analysed on printouts by C B-L. The number of false negative (missed) ventricular extrasystoles with this approach is negligible. ${ }^{25}$ In three recordings with frequent ventricular extrasystoles ( $>100$ in 24 hours), the entire 24 hour electrocardiogram was printed out on paper and the beats counted manually. Premature normal events (that is those that occurred earlier than $30 \%$ of the preceding $\mathbf{R} R$ interval were defined as atrial extrasystoles) were detected on an uninterrupted replay. ${ }^{24}$ This setting has been found empirically to give the best balance between the risk of missing atrial extrasystoles (false negatives) and noting false positives. ${ }^{25}$ All premature normal events detected by the analyser were visually validated by the operator, who thereby minimised the number of false positive atrial extrasystoles. The electrocardiographic criteria for classification of extrasystoles were those of Friedman. ${ }^{26} \mathrm{~A}$ pause was defined as an $R R$ interval lasting $>2000 \mathrm{~ms}$; compensatory pauses that followed premature QRS complexes were excluded.

We measured the mean 24 hour heart rate as the mean number of heart beats/min estimated from the total number of beats in 24 hours. The minimal heart rate was the smallest number of beats in one minute during a 24 hour period. When the normal limits for the mean 24 hour heart rate and for minimal heart rate were calculated we took into account the additive effects of age, sex, smoking, and physical activity. ${ }^{27}$ The patient's participation in leisure time physical activity and their smoking habits were classified as described earlier. ${ }^{27}$

\section{STATISTICAL ANALYSIS}

Values are expressed as mean (1 SD) unless otherwise stated. Pearson's coefficient of correlation was used to quantify the degree of relation between QT intervals in leads II and V4 and to assess the relation between electrocardiographic variables and serum concentrations of potassium and magnesium.

\section{Results}

No patient had a history of cardiovascular disease. Four patients reported infrequent palpitation. No $\stackrel{\text { ? }}{\rightarrow}$ patient had experienced faintness or syncope. Onec patient had chronic obstructive lung disease and $\overline{\bar{n}}$. diabetes mellitus (case 8). The physical examination was normal in all other patients.

\section{ELECTROLYTES}

The mean serum concentrations of potassium andmagnesium were $2.7(0.3) \mathrm{mmol} / \mathrm{l}$ and $0.6(0.1) \vec{\omega}$ mmol/1 respectively (table 1 ). Fifteen patients had hypomagnesaemia. The serum concentration of cal-? cium was normal in every patient. There was nolinear relation between the measured electrocar $-i$ diographic variables and the serum concentrations of potassium or magnesium.

\section{ELECTROCARDIOGRAPHIC FINDINGS}

The $P Q$ interval was normal in all patients and the mean value was $0.16(0.02) \mathrm{s}$ (table 1$)$. Mean $Q R S \stackrel{\text { S. }}{\text { - }}$ interval was $0.09(0.01) \mathrm{s}$; the interval was slightly $\vec{\bullet}$ prolonged in one patient (table 1 ).

Depression of the ST segment of $\geqslant 0.5 \mathrm{~mm}$ was found in seven patients, flat or low amplitude $T_{O}$ waves in seven, and $U$ wave amplitudes of $\geqslant 1 \mathrm{~mm}$ in three patients (table 1). The ST-T and $U$ waveo changes were generally slight (fig 1). By theseo electrocardiographic criteria seven patients had $a \circ$ pattern that was compatible with hypokalaemia $\overrightarrow{\overrightarrow{0}}$ (table 1). No patient showed a pattern that was 3 typical of hypokalaemia on the resting electrocardiogram.

The QTE interval (onset of QRS to end of T wave) was measurable in both leads in all but two patientso and the QTtan in all but three patients, in whom there was fusion with the $U$ wave or the following $P$ S wave. All except two patients (cases 4 and 7) showedo prolongation of the QTE and/or QTtan intervals (table 1). Figure 2 shows the group mean values of theo $\mathrm{QT}$ intervals. Individual discrepancies of $\mathrm{QT}^{-}$ measurements between the two leads were related too the presence of superimposed $U$ waves or delayed repolarisation changes.

\section{HOLTER MONITORING}

Nine $(45 \%)$ patients had one or more ventricular extrasystoles in the 24 hour Holter recording (table 2). Two patients (cases 1 and 3) had more than 200 ventricular extrasystoles per 24 hour and one (case 7 , had more than 1000 . In both patients these were asymptomatic and multiform with more than two QRS configurations, and in one of them they: frequently occurred in pairs and in bigeminy. Of the remaining seven patients with ventricular extrasys $-\frac{0}{0}$ 


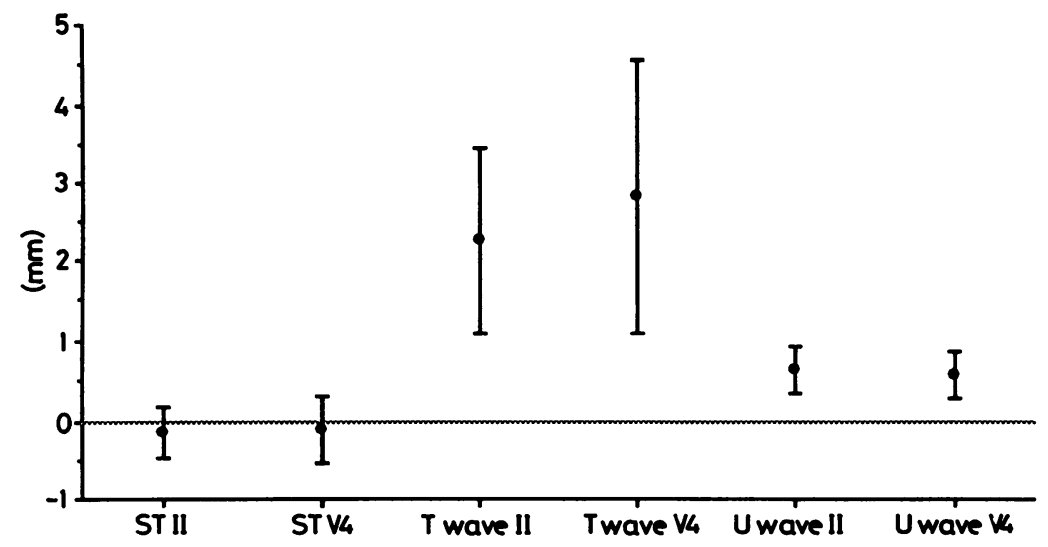

Fig 1 Mean (1 SD) ST segment deviations and $T$ and $U$ wave amplitudes in leads II and V4. Note the relation between the $T$ wave amplitude and the $U$ wave amplitude.

toles, one patient had two different QRS configurations, two had a single episode of $R$ on $T$ phenomenon, but none of them had pairs or episodes with bigeminy, trigeminy, or quadrigeminy. No patient had ventricular tachycardia.

Nine $(45 \%)$ patients had one or more atrial extrasystoles in 24 hours, but only one patient (case 15) had more than 200 in 24 hours (table 2). One patient had a single episode of supraventricular tachycardia (case 20). The mean 24 hour heart rate was 79 (9) beats $/ \mathrm{min}$ (range $62-90$ beats $/ \mathrm{min}$ ). When corrected for age, sex, smoking habits, and physical activity the calculated mean 24 hour heart rate was higher than the predicted normal values in two patients (cases 15 and 18). In case 15 this may have been related to frequent atrial extrasystoles. The mean minimal heart rate was 56 (9) beats/min (range 41-70 beats/min). It was normal in all except two patients (cases 3 and 5), in whom it was lower than the predicted normal values. No patient had pauses $(R R$ interval $>2.0 \mathrm{~s}$ ).

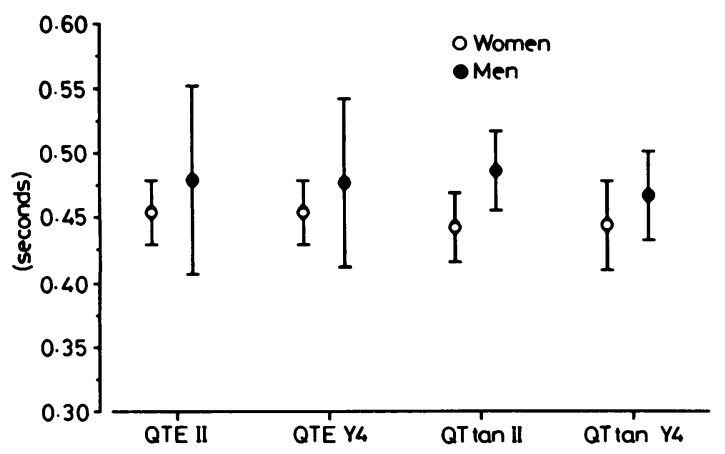

Fig 2 Mean (1 SD) QTE and QTtan intervals in women and in men. The $Q T$ intervals are corrected for heart rate.

\section{Discussion}

Bartter's syndrome offers a unique opportunity to study clinical effects of chronic electrolyte disturbances. These patients have persistent, probably lifelong, hypokalaemia, often subnormal extracellular magnesium concentrations, ${ }^{18}$ and no apparent structural heart disease.

In most of our patients mean and minimal heart rates were normal after correction for smoking habits, age, and physical activity. In both experimental and clinical studies there is disagreement about the effect of low potassium concentration on the rate of the sinoatrial node ${ }^{928}$ but it is generally stated to depress the heart rate. ${ }^{28}$

Only one patient had signs of conduction disturbances on the resting electrocardiogram or during 24 hour Holter monitoring. The $P Q$ interval was normal in each patient and the QRS interval was also normal in all except one patient. Several experimental studies have documented a delay or block of atrioventricular conduction as the extracellular concentrations of potassium are lowered, ${ }^{29}$ and a diffuse depression of intraventricular conduction in more advanced hypokalaemia. ${ }^{9}$ In contrast with the high frequency

Table 2 Number of extrasystoles during 24 hour Holter monitoring in 20 patients with Bartter's syndrome

\begin{tabular}{lcc}
\hline No of extrasystoles in 24 hours & 'ES & AES \\
& No $(\%)$ & No $(\%)$ \\
\hline None & $11(55)$ & $11(55)$ \\
$\geqslant 1$ & $9(45)$ & $9(45)$ \\
$>10$ & $5(25)$ & $3(15)$ \\
$>100$ & $3(15)$ & $2(10)$ \\
$>200$ & $2(10)$ & $1(5)$ \\
$>1000$ & $1(5)$ & 0
\end{tabular}

VES, ventricular extrasystoles; AES, atrial extrasystoles. 
of conduction defects in experimentally induced hypokalaemia, prolongation of the $P Q$ or $Q R S$ intervals has been rarely seen in clinical hypokalaemia. ${ }^{30}$

In clinical hypokalaemia the most consistent electrocardiographic changes are the appearance of a prominent $U$ wave, depression of the ST segment, and decreased amplitude of the $T$ wave. ${ }^{8230}$ In our patients a pattern "typical" of hypokalaemia was not seen in any individual and a pattern "compatible" with hypokalaemia was seen in only a few. These patterns were more common in patients with other diseases who had comparable serum concentrations of potassium. ${ }^{823}$ Moreover, the magnitude of the ST segment depressions and the deviations in the amplitude of $T$ and $U$ waves were less pronounced in our patients. ${ }^{30}$

In most of our patients the QT interval was prolonged. The relation of the QT interval to the serum concentration of potassium is controversial. Some have suggested that the QT interval is prolonged by hypokalaemia ${ }^{30}$ and others that it is normal or actually shortened. ${ }^{2123}$ Because there are several potential sources of inaccuracy in measuring the QT intervals in the clinical setting ${ }^{31}{ }^{32}$ it is difficult to evaluate the biological significance of minor QT changes and to use the QT interval as a reliable reflection of repolarisation changes. ${ }^{33}$ It is, however, well known that a decrease in extracellular potassium concentrations affects repolarisation by prolonging the action potential duration..$^{6811}$

The slight general electrocardiographic changes in our patients contrasted with those in previous studies. $^{230}$ Although changes on the electrocardiogram have usually been related to abnormalities in the extracellular electrolyte concentrations, it is the altered potassium gradient across the cell membrane that induces changes on the electrocardiogram. ${ }^{8}$ In acute experiments, intracellular potassium concentrations are relatively stable and altered potassium gradients are largely caused by the changes in extracellular concentrations of potassium. In our patients, however, the intramyocardial potassium concentration, and thus also the gradient across the myocardial cell membrane, is unknown. There are, however, a multitude of factors (see below) that make it difficult to compare electrocardiographic and electrophysiological changes in various states of hypokalaemia.

Supraventricular and ventricular tachycardia seemed to be no more common than in healthy subjects. ${ }^{25}{ }^{34}$ All except two of our patients had a normal 24 hour Holter recording according to the recently suggested normal limit for the number of atrial and ventricular extrasystoles in 24 hours, ${ }^{25}$ that is 200 extrasystoles in $\mathbf{2 4}$ hours. The significance of the single episodes of $R$ on $T$ phenomenon seen in $\frac{2}{7}$ two other patients with less than 200 ventricular $c$. extrasystoles in 24 hours was hard to evaluate $\Rightarrow$ because most of the complex extrasystoles can occur? as incidental findings in a few healthy subjects. ${ }^{25}{ }^{34} \mathrm{In}_{\mathrm{C}}$ previous studies patients with hypokalaemia hado more extrasystoles than a control hospital $\frac{\bar{s}}{\sigma}$ population. ${ }^{130}$ Studies with microelectrodes in rab- $\triangle$ bits showed that continued perfusion with solutions containing low concentrations of potassium leads to supraventricular and ventricular extrasystoles; ectopic tachycardia and, finally, ventricular $\vec{\omega}_{\omega}$ fibrillation.9 The tendency for extrasystoles to 9 develop during hypokalaemia has been attributed to several electrophysiological effects, including an enhanced automaticity, a prolonged phase 3 of thei action potential duration, and an enhanced reentry caused by depressed conduction. ${ }^{12}$

The type of rhythm disorder induced or abolishec by changes in the serum concentration of potassium depends not only on the extracellular potassium concentration but also on other factors, such as thes rapidity with which the potassium concentration changes, the sensitivity of the various cardiac fibre $s_{0}^{\circ}$ to such changes, the extracellular concentration of other electrolytes, the underlying state of the myocardium, the interactions with neurogenic s factors, and cardioactive drugs. ${ }^{815}$ Moreover, studies in rabbits showed that cardiac muscle was protecteकी against loss of potassium during chronic potassium depletion by an adaptive increase in the density of sodium pump receptors. ${ }^{35}$ It is not yet known whether these results can be extrapolated to humang beings.

We found that although extracellular concentrations of potassium were usually very low in patients with Bartter's syndrome, the electrocardiographic changes were slight and arrhythmias were not common. Further studies will be needed to show whether these findings are relevant to the long term? prognosis.

This study was supported by the Swedish Nationat Association against Heart and Chest Disease and the Medical Society of Gothenburg.

\section{References}

1 Davidson S, Surawicz B. Ectopic beats and atrio주 ventricular conduction disturbances in patients witho hypopotassemia. Arch Intern Med 1967;120:280-5.

2 Hulting J. In-hospital ventricular fibrillation and itș relation to serum potassium. Acta Med Scand 1081;647:109-16.

3 Holland OB, Nixon JV, Kuhnert I. Diuretic-induced ventricular ectopic activity. Am J Med 1981;70? 762-8. 
4 Papademetriou V. Diuretics, hypokalemia, and cardiac arrhythmias: a critical analysis. Am Heart J 1985;111:1217-24.

5 Dyckner T, Wester PO. Ventricular extrasystoles and intracellular electrolytes in hypokalemic patients before and after correction of the hypokalemia. Acta Med Scand 1978;204:375-9.

6 Surawicz B, Lepeschkin E, Herrlich HC, Hoffman BF. Effect of potassium and calcium deficiency on monophasic action potential, electrocardiogram and contractility of isolated rabbit hearts. Am J Physiol 1959;196:1302-7.

7 Deleze J. Perfusion of a strip of mammalian ventricle. Effects of K-rich and Na-deficient solutions on transmembrane potentials. Circ Res 1959;7:461-5.

8 Surawicz B. Relationship between electrocardiogram and electrolytes. Am Heart J 1967;73:814-34.

9 Gettes LS, Surawicz B, Shiue JC. Effect of high K, low $K$, and quinidine on $Q R S$ duration and ventricular action potential. Am J Physiol 1962;203:1135-40.

10 Hoffman BF, Suckling EE. Effect of several cations on transmembrane potentials of cardiac muscle. Am J Physiol 1956;186:317-24.

11 Gettes L, Surawicz B. Effects of low and high concentrations of potassium on the simultaneously recorded Purkinje and ventricular action potentials of the perfused pig moderator band. Circ Res 1968;23:717-29.

12 Commerford PJ, Lloyd EA. Arrhythmias in patients with drug toxicity, electrolyte, and endocrine disturbances. Med Clin North Am 1984;68:1051-78.

13 Fisch C. Relation of electrolyte disturbance to cardiac arrhythmias. Circulation 1973;47:408-19.

14 Gettes LS. Possible role of ionic changes in the appearance of arrhythmias. Pharmacol Ther B 1976;2:787-810.

15 Watanabe Y, Dreifus LS. Electrophysiological effects of magnesium and its interactions with potassium. Cardiovasc Res 1972;6:79-88.

16 Bartter FC, Pronove P, Gill JR, MacCardle RC. Hyperplasia of juxtaglomerular complex with hyperaldosteronism and hypokalemic alkalosis. $\mathrm{Am} \mathrm{J}$ Med 1962;33:811-28.

17 Gill JR. Bartter's syndrome. Annu Rev Med 1980;31:405-19.

18 Rudin A. Bartter's syndrome. A review of 28 patients followed for ten years. Acta Med Scand 1988;224:165-71.

19 Rose GA, Blackburn H, Gillum RF, Prineas RJ. Classification of the electrocardiogram for population studies. Geneva: WHO Monographic series 1982:123-68.
20 Blomström-Lundqvist C, Jonsson R, Edvardsson $\mathrm{N}$. Ventricular repolarization time-effect of chronic beta blockade with class II and/or class III properties. Double-blind cross-over study using transesophageal atrial pacing. Am J Noninvas Cardiol 1988;2:32-7.

21 Lepeschkin E, Surawicz B. The measurement of the QT interval of the electrocardiogram. Circulation 1952;6:378-88.

22 Bazett HC. An analysis of the time-relations of electrocardiograms. Heart 1920;7:353-70.

23 Surawicz B, Braun HA, Crum WB, Kemp RL, Wagner $S$, Bellet S. Quantitative analysis of the electrocardiographic pattern of hypopotassemia. Circulation 1957;16:750-63.

24 Bjerregaard $P$. The quality of ambulatory ECGrecordings and accuracy of semi-automatic arrhythmia analysis. An evaluation of the MedilogPathfinder system. Eur Heart J 1980;1:417-25.

25 Bjerregaard P. Premature beats in healthy subjects 4079 years of age. Eur Heart $J$ 1982;3:493-503.

26 Friedman HH, ed. Diagnostic electrocardiography and vector-cardiography. New York: McGraw-Hill, 1971:383-585.

27 Bjerregard P. Mean 24 hour heart rate, minimal heart rate and pauses in healthy subjects $40-79$ years of age. Eur Heart J 1983;4:44-51.

28 Fisch C, Knoebel SB, Feigenbaum H, Greenspan K. Potassium and the monophasic action potential, electrocardiogram, conduction and arrhythmias. Prog Cardiovasc Dis 1966;8:387-418.

29 Paes de Carvalho A, Langan WB. Influence of extracellular potassium levels on atrioventricular transmission. Am J Physiol 1963;205:375-81.

30 Weaver WF, Burchell HB. Serum potassium and the electrocardiogram in hypokalemia. Circulation 1960;21:505-21.

31 Cowan JC, Yusoff K, Moore M, et al. Importance of lead selection in QT interval measurement. $A m J$ Cardiol 1988;61:83-7.

32 Sylvén JC, Horacek BM, Spencer CA, Klassen GA, Montague TJ. QT interval variability on the body surface. J Electrocardiol 1984;17:179-88.

33 Seed WA, Noble MIM, Oldershaw P, et al. Relation of human cardiac action potential duration to the interval between beats: implications for the validity of rate corrected QT interval (QTc). Br Heart $J$ 1987;57:32-7.

34 Clarke JM, Hamer J, Shelton JR, Taylor S. The rhythm of the normal human heart. Lancet 1976;iv:508-12.

35 Ward JPT, Cameron IR. Adaptation of the cardiac muscle sodium pump to chronic potassium deficiency. Cardiovasc Res 1984;18:257-63. 